\title{
PLACE ET LIMITES DE LA CHIRURGIE ENDONASALE DANS LE TRAITEMENT DES MUCOCELES SINUSIENNES
}

\author{
I. HARIGA, S. ZRIBI, K. KHAMASSI, M. BEN AMOR, A. BOUZAÏANI, O. BEN GAMRA, \\ CH. CHAMMAKHI ${ }^{*}, \mathrm{CH}$. MBAREK, A. EL KHEDIM \\ SERVICE D'ORL ET DE CHIRURGIE CERVICO-FACIALE \\ *SERVICE DE RADIOLOGIE \\ HÔPITAL HABIB THAMEUR, TUNIS, TUNISIE
}

\begin{abstract}
Objectif : Préciser les indications et les limites de la chirurgie endonasale dans le traitement des mucocèles sinusiennes. Matériel et méthodes : Il s'agit d'une étude rétrospective à propos de 15 patients colligés sur 13 ans (1994-2006). Tous les patients ont bénéficié d'une endoscopie nasale et d'une TDM du massif facial. Une IRM a été pratiquée dans 10 cas. Le traitement chirurgical comportait une marsupialisation ou une exérèse de la mucocèle. Les voies d'abord étaient endonasales, externes ou combinées, en fonction de l'extension ou de la topographie des lésions. La surveillance en postopératoire a été clinique et radiologique.

Résultats : La TDM a montré une atteinte ethmoïdo-frontale ( 6 cas), maxillaire ( 5 cas), ethmoïdale isolée ( 2 cas), frontale isolée (1 cas) et sphénoïdale (1 cas). L'IRM a montré une extension orbitaire dans 7 cas et endocrânienne dans 3 cas. La voie d'abord chirurgicale était endonasale exclusive dans 12 cas. Une voie combinée (endonasale et externe) a été nécessaire chez un patient ayant une atteinte frontale isolée et un autre ayant une atteinte ethmoïdo-frontale. Un patient ayant une mucocèle maxillaire latérale a été opéré par voie de Caldwell-Luc. Aucune récidive n'a été notée avec un recul moyen de 14 mois.

Conclusion : La chirurgie endonasale représente la technique de choix dans le traitement des mucocèles sinusiennes et donne de bons résultats à long terme. Le siège et l'extension des mucocèles représentent les principaux facteurs déterminants dans le choix de la voie d'abord.
\end{abstract}

Objective : To determine indications and limits of endonasal approach for the treatment of sinusal mucoceles.

Materials and methods : We carry a retrospective study about 15 patients treated between 1994 and 2006. All patients had had nasal endoscopy and sinonasal CT. MRI was performed in 10 cases. Surgical treatment included marsupialization or mucocele excision. Either endonasal, external or combined approaches were performed, according to the extension or the localization of the mucocele. Postoperative follow-up was clinical and radiological.

Results : CT showed that mucoceles were ethmoido-frontal (6 cases), maxillary (5 cases), ethmoidal (2 cases), frontal ( 1 case) and sphenoidal (1 case). MRI showed orbital (7 cases) and endocranial extension (3 cases). Surgical approach was exclusively endonasal in 12 cases. combined approach (endonasal and external) was necessary for one patient having frontal mucocele and another having ethmoido-frontal one. One patient having lateral maxillary mucocele was operated by Caldwell-Luc approach. No recurrences were noted after a mean follow-up period of 14 months.

Conclusion : Endoscopic endonasal surgery is the technique of choice for the treatment of sinusal mucoceles and provides good long-term results. Localization and extension of the mucoceles are the most important factors in the choice of the surgical approach.

\section{INTRODUCTION}

Les mucocèles sont des formations bénignes pseudokystiques se développant au niveau des sinus de la face, dont la paroi est constituée par une muqueuse plus ou moins modifiée de la cavité sinusienne et le contenu est un liquide épais et aseptique. Leur physiopathologie implique une inflammation muqueuse associée à une obstruction ostiale. La chirurgie naso-sinusienne et les antécédents de traumatisme constituent les principaux facteurs favorisant leur survenue. Leur diagnostic est orienté par la clinique et l'imagerie et leur traitement repose sur la chirurgie. Actuellement, la marsupialisation par voie endonasale constitue le traitement de choix $(1,2,3,4)$. II s'agit d'une procédure non invasive, sans séquelles esthétiques, qui respecte l'architecture des sinus et leurs sites naturels de drainage (1). Les voies externes permettant une exérèse complète des mucocèles sont devenues d'indications plus rares.

Le but de ce travail est de préciser les indications de la chirurgie endonasale dans les mucocèles sinusiennes et de discuter les limites de cette voie en fonction du siège et de l'extension des lésions.

\section{MATERIEL ET METHODES}

II s'agit d'une étude rétrospective à propos de 15 patients suivi et traités sur 13 ans (1994-2006) et ayant une mucocèle sinusienne. Tous les patients ont bénéficié d'une endoscopie nasale et d'une TDM du massif facial. Une IRM a été pratiquée dans 10 cas. Le traitement chirurgical comportait une marsupialisation ou une exérèse de la 
mucocèle. Les voies d'abord étaient endonasales, externes ou combinées, en fonction de l'extension ou de la topographie des lésions. En cas de marsupialisation, les adhérences périostées orbitaires et méningées ont été respectées. L'examen anatomopathologique de la paroi réséquée confirmait le diagnostic de mucocèle dans tous les cas. La surveillance en postopératoire a été clinique et radiologique avec un recul moyen de 14 mois (9-26 mois).

\section{RESULTATS}

L'âge moyen était de 42 ans (8-81 ans) et le sex-ratio de 2. Sept patients avaient des antécédents de chirurgie endonasale (ethmoïdectomie pour polypose naso-sinusiene chez 5 patients, Caldwell-Luc chez un patient et méatotomie moyenne chez un autre). Deux patients avaient une rhinosinusite chronique et un autre avait des antécédents de traumatisme nasal nécessitant une réduction des os propres du nez.

\begin{tabular}{|l|c|}
\hline Signes fonctionnels & Nombre de cas \\
\hline Signes rhinologiques: & 7 \\
Obstruction nasale & 6 \\
Rhinorrhée & 3 \\
Hyposmie & \\
\hline Signes ophtalmologiques: & 6 \\
(Edème palpébral & 2 \\
Diplopie & 2 \\
Chute de l'acuité visuelle & 1 \\
\hline Ptosis & 11 \\
\hline Algies faciales & \\
\hline
\end{tabular}

Tableau I: Symptomatologie fonctionnelle

Les algies faciales, l'obstruction nasale, la rhinorrhée et l'œdème palpébral étaient les signes fonctionnels les plus fréquents (Tableau I). L'examen clinique trouvait une déformation de la face (5 cas), une exophtalmie (5 cas) et/ou un bombement au niveau du méat moyen ( 2 cas). La TDM a montré une atteinte ethmoïdo-frontale ( 6 cas), maxillaire ( 5 cas dont une atteinte bilatérale, Fig 1$)$, ethmoïdale ( 2 cas, Fig 2$)$, frontale ( 1 cas, Fig 3 ) et sphénoïdale (1 cas). L'IRM a objectivé une extension orbitaire dans 7 cas et endocrânienne dans 3 cas (Fig 4)

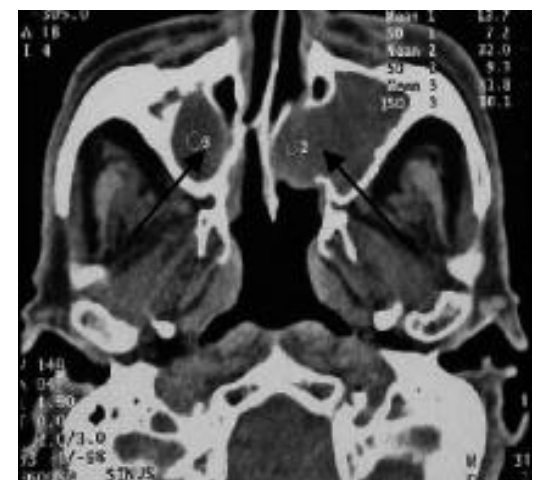

Fig. 1 : TDM (coupe axiale) : Mucocèle maxillaire bilatérale

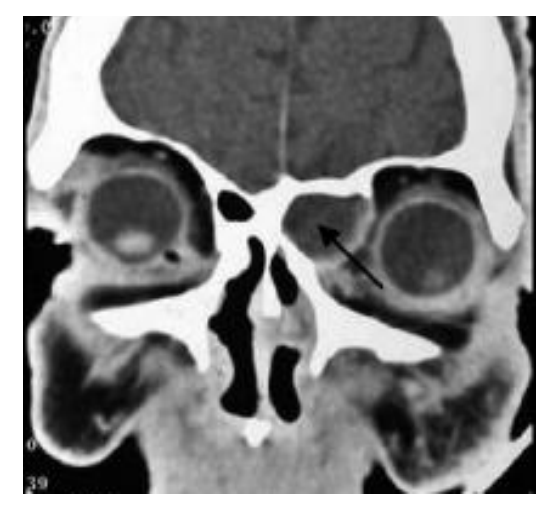

Fig. 2 : TDM (coupe coronale) : Mucocèle ethmoïdale gauche

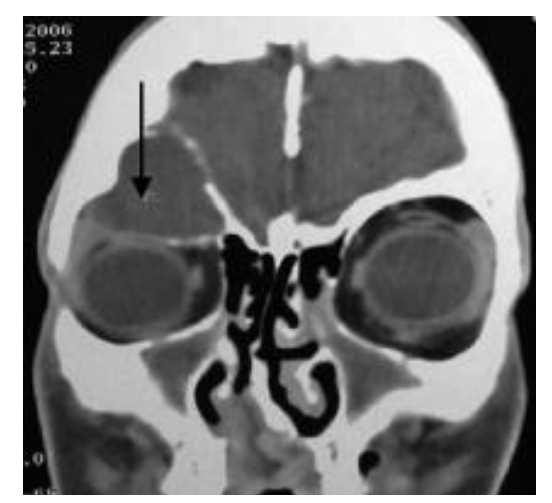

Fig 3 : TDM (coupe coronale) : Mucocèle frontale droite

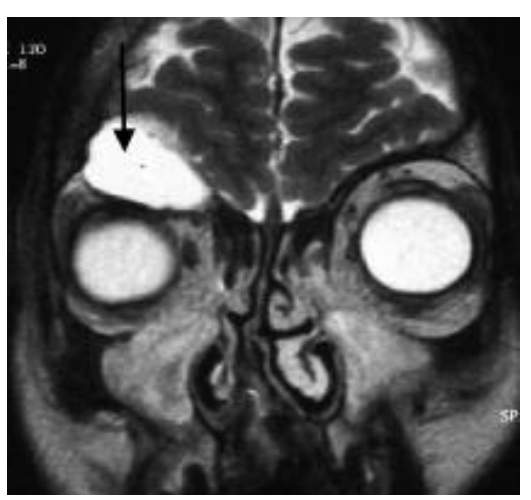

Fig 4 : IRM en T2 (coupe coronale) :

Mucocèle frontale droite avec extension orbitaire et endocrânienne

La voie d'abord chirurgicale était endonasale exclusive dans 12 cas, vestibulaire dans 1 cas et combinée dans 2 cas (Tableau II). Trois patients ont nécessité donc un abord externe devant une atteinte sinusienne (frontale ou maxillaire) très latéralisée et dont l'accès par voie endoscopique était difficile. Parmi ces patients, deux ont été opéré par voie combinée (mucocèle frontale et ethmoïdofrontale) et un autre par voie de Caldwell-Luc (mucocèle maxillaire latérale).

Une marsupialisation a été pratiquée chez les 12 patients 
opérés par voie endonasale. L'accès aux mucocèles ethmoïdo-frontaux a nécessité une ethmoïdectomie. Pour les mucocèles maxillaires, une méatotomie moyenne a été réalisée pour accéder à la poche mucocélique. L'exérèse totale de la mucocèle a été réalisée dans les 3 cas où la voie externe a été nécessaire. Aucun incident peropératoire n'a été noté. L'évolution en postopératoire a été favorable dans tous les cas avec régression de la symptomatologie fonctionnelle. Une TDM de contrôle a été réalisée chez cinq patients et a montré l'absence de récidive dans tous les cas (Fig 5). Par ailleurs, la surveillance endoscopique n'a pas montré de récidive avec un recul moyen de 14 mois.

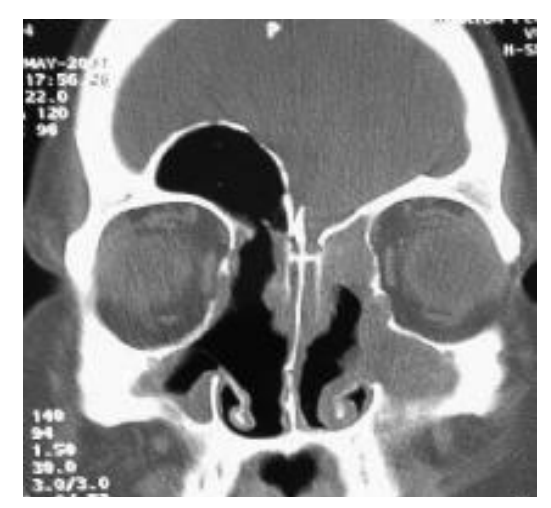

Fig. 5 : TDM (coupe coronale) :

Aspect postopératoire après marsupialisation d'une mucocèle ethmoïdo-frontale droite

\begin{tabular}{|c|c|c|c|c|}
\hline $\begin{array}{c}\text { Sinus atteints } \\
\text { et voies d'abords }\end{array}$ & Endonasale & Combinée & Externe & Total \\
\hline Ethmoïdo-frontal & 5 & 1 (endonasale+coronale) & 0 & 6 \\
Maxillaire & 4 & 0 & 1 (Caldwell-Luc) & 5 \\
Ethmoïdal & 2 & 0 & 0 & 2 \\
Frontal & 0 & 1 (endonasale+sourcilière) & 0 & 1 \\
Sphénoïdal & 1 & 0 & 0 & 1 \\
\hline Total & 12 & 2 & 1 & 15 \\
\hline
\end{tabular}

Tableau II: Sinus atteints et voies d'abord

\section{DISCUSSION}

La mucocèle sinusienne est une affection rare touchant surtout l'adulte de la cinquième décade (2). La chirurgie naso-sinusienne en constitue le facteur étiopathogénique le plus fréquent. En effet, des antécédents de chirurgie endonasale sont retrouvés jusqu'à dans 45\% (46,6\% dans notre étude) et d'intervention de Caldwell-Luc jusqu'à $50 \%$ des cas $(1,2,5)$. Cette origine iatrogène est expliquée par la fermeture du canal naso-frontal par un processus cicatriciel, la présence de synéchies ethmoïdales antérieures, la présence de reliquats d'apophyse unciforme ou la fermeture ostiale fibreuse. Tous ces facteurs entraînent une obstruction sinusienne qui favorise la formation des mucocèles (6). Par ailleurs, les trauma- tismes faciaux, les rhinosinusites chroniques et les polyposes peuvent être incriminés.

La stratégie thérapeutique concernant les mucocèles dépend essentiellement du siège et de l'extension des lésions, déterminées sur les données de l'imagerie. En effet, la TDM permet de préciser l'étendue des lésions dans les différents sinus et de rechercher une lyse osseuse, une atteinte orbitaire ou endocrânienne. L'IRM, non systématique, permet de mieux évaluer l'extension endocrânienne et orbitaire et de distinguer la mucocèle du processus rétentionnel sinusien qui l'accompagne.

La chirurgie des mucocèles a connu ces dernières années un essor important avec l'avènement de la chirurgie endonasale. Le concept de l'exérèse totale de la paroi mucocélique réalisée lors d'un abord externe est modifié au profit du principe de la marsupialisation dans les fosses nasales avec l'évolution des connaissances concernant la pathogénie des mucocèles et le risque majeur que revêt l'exérèse totale de la paroi souvent en rapport intime avec les organes de voisinage $(7,8,9)$. La simple marsupialisation par voie endonasale laisse une paroi mucocélique qui n'est autre que la muqueuse sinusienne plus ou moins modifiée de façon réversible par l'inflammation et l'hypersécrétion (4). Par ailleurs, l'os déminéralisé par la mucocèle se reconstitue après marsupialisation.

La voie endonasale est la technique de choix en cas d'atteinte ethmoïdale ou ethmoïdo-frontale, qui représente $80 \%$ de l'ensemble des mucocèles (10). Parfois, une méatotomie moyenne ou une ethmoïdectomie sont nécessaires pour accéder à la poche mucocélique. Cette voie est actuellement privilégiée par rapport aux voies d'abord trans-faciales ou sous labiales qui étaient largement utilisées auparavant (Caldwell-Luc, Degloving, coronale, para-latéro-nasale). L'atteinte frontale latérale impose souvent une voie externe (sourcilière ou coronale) ou combinée, en cas d'inaccessibilité par voie endonasale à la poche mucocélique, même après résection du plancher du sinus frontal $(11,12,13)$. Ce contrôle par voie externe des mucocèles latéralisées permettra même d'avoir de meilleurs résultats en matière de récidive que la voie endonasale exclusive (0\% contre $25 \%$ ) (14).

En cas d'extension vers l'orbite, les voies endonasale ou combinée peuvent être préconisées, permettant une régression totale des signes ophtalmologiques (3). En cas d'extension endocrânienne, l'abord endonasal peut aussi être réalisé. La voie neurochirurgicale est réservée aux cas où il existe une déhiscence osseuse avec dénudation de la dure-mère.

Dans notre série, 8 patients ayant une mucocèle ethmoïdale ou ethmoïdo-frontale ont été opérés par voie endonasale, associée à une voie coronale dans 1 cas. Un patient ayant une atteinte frontale isolée a été opéré par voie de Jacques. Aucune récidive n'a été notée chez tous ces patients. Ces résultats rejoignent celles de la majori- 
té des auteurs où le taux de récidive est quasi-nul $(1,4,14)$.

Les mucocèles maxillaires représentent environ $20 \%$ de l'ensemble des mucocèles $(2,6)$. Elles peuvent être traitées par une marsupialisation par voie endonasale avec de bons résultats à long terme $(15,16)$. Cependant, la localisation au niveau de la partie antérieure ou latérale du sinus maxillaire est souvent inaccessible par la voie de méatotomie moyenne et impose une voie de Caldwell-Luc associée ( 1 cas dans notre étude sur les 5 patients ayant une atteinte maxillaire). Les complications de cette voie sont représentées essentiellement par les hypoesthésies ou paresthésies jugales et labiales en rapport avec une atteinte du nerf infra-orbitaire.

Les mucocèles ethmoïdales postérieures et sphénoïdales sont beaucoup plus rares: 1 seul cas dans notre étude et 1 à $3 \%$ de l'ensemble des mucocèles $(3,17,18)$. Leur traitement par voie endonasale constitue la technique de choix et donne de bons résultats à long terme avec un faible taux de récidive $(1,14)$.

La mucocèle fistulisée à la peau, la marsupialisation insuffisante et le doute diagnostique malgré un bilan un bilan radiologique poussé constituent aussi une limite à la voie endonasale et imposent une voie trans-faciale ou externe $(8,9,19)$. Ces situations se voient essentiellement dans la chirurgie des récidives mucocéliques ou dans les mucocèles secondaires à une chirurgie de tumeur sinusienne où l'architecture anatomique endonasale est totalement perturbée exposant le chirurgien à des difficultés par une approche endonasale exclusive.

\section{CONCLUSION}

Bien qu'elle soit impliquée dans la pathogénie des mucocèles sinusiennes, la chirurgie endonasale est devenue actuellement la technique de choix dans leur traitement. Outre son avantage sur le plan esthétique, elle constitue une chirurgie fonctionnelle qui respecte la physiologie de la muqueuse sinusienne et dont la morbidité est moindre par rapport aux voies externes classiques. Néanmoins, ses indications doivent être bien posées et ses limites doivent être connues. Par ailleurs, elle nécessite une maîtrise parfaite de la technique opératoire et une connaissance de l'anatomie endoscopique endonasale. Ceci permettra d'éviter les complications de cette chirurgie et d'avoir de meilleurs résultats à long terme en matière de récidives.

\section{REFERENCES}

(1) Serrano E, Klossek JM, Percodani J, Yardeni E, Dufour X. Surgical management of paranasal sinus mucoceles: A long-term study of 60 cases. Otolaryngol Head Neck Surg 2004;131:133-140.

(2) Koobaa J, Kharrat S, Jendoubi N, Ben Azzouna H, Zribi S, Mbarek Ch, El Khedim A. Les mucocèles sinusiennes: A propos de 9 cas. J Tun ORL 2001;7:25-30

(3) Hazan A, Blin P, Peytral C, Cymbalista M, Chevalier E, Laroche A, Bego B, Bodard-Rickelman E, Sener Y, Delval A. A propos de deux cas de mucocèle avec complications ophtalmologiques majeures traitées par chirurgie endonasale endoscopique. Ann Otolaryngol Chir Cervicofac 1996;113:92-98.

(4) Raji A, Essaadi M, Detsouli M, Kadiri F, Laraqui N, Touhami M, Chekkoury Idrissi A, Benchakroun Y. La chirurgie endonasale: résultats dans la chirurgie des mucocèles ethmoïdo-frontales. Rev Laryngol Otol Rhinol 2000;121:251-254.

(5) Rivron A, Bourdinière J. Mucocèles et pneumosinus dilatans. Editions techniques. Encycl Med Chir (Paris, France), Oto-rhino-laryngologie 20-465-A-10, 12, 1990, $12 \mathrm{p}$.

(6) Raynal M, Peynegre R, Beautru R, Coste A. Mucocèles sinusiennes et iatrogénie chirurgicale. Ann Otolaryngol Chir Cervicofac 1999;116:85-91.

(7) Benninger MS, Marks S. The endoscopic management of sphenoid and ethmoid mucoceles with orbital and intranasal extension. Rhinology 1995;33:157161.

(8) Naudo P, Gilain L, Cost A, Lebievre G, Peynegre R. Chirurgie fonctionnelle endoscopique des mucocèles sinusiennes. Ann Otolaryngol Chir Cervicofac 1994;111:23-27.

(9) Serrano E, Pessey JJ, Lacomme Y. Sinus mucoceles: diagnosis and surgical aspects (report of 8 cases treated with rhinosurgical endoscopy). Acta
Otolaryngol Belg 1992;46:287-292

(10) Fourgeront B, Lamas G. Mucocèles frontales et ethmoïdales - A propos de 17 cas. Ann Otolaryngol Chir Cervicofac 1990;107:323-327

(11) Close LG, Leach JL, Lee NK, et al. Endoscopic resection of the intranasal frontal sinus floor. Ann Otol Rhinol Laryngol 1994;103:952-958.

(12) May M. Frontal sinus surgery. Operative Tech Otolaryngol Head Neck Surg 1995;6:184-192.

(13) Schmerber S, Cuisnier O, Delalande C, Verougstraete G, Reyt E. Surgical strategy in paranasal sinus mucoceles. Rev Laryngol Otol Rhinol 2002;123:9397.Shmerber S, Cuisnier O, Delalande C, Verougstraete G, Reyt E.e endonasale exclusive ( $0 \%$ contre $25 \%$ ) [

(14) Venail F, Marlier F, Kakeieff M, Garrel R, Rhombdane S, Guerrier B, Crampette L. Combined approach (endoscopic and external) for the treatment of sinusal mucoceles. Rev Laryngol Otol Rhinol 2003;124:165-170.

(15) Saito T, Ikeda T, Kono Y, Ohtsubo T, Noda I, Saito H. Implications of endoscopic endonasal surgery for the treatment of postoperative maxillary mucoceles. Otorhinolaryngol J Relat Spec 2000;62:43-48.

(16) Busaba NY, Salman SD. Maxillary sinus mucoceles: clinical presentation and long-term results of endoscopic surgical treatment. Laryngoscope 1999;109:1446-1449.

(17) Barat JL, Marchal JC, Bracard S, et al. Mucoceles of the of the sphenoid sinus. J Neuroradiol 1990;17:135-151.

(18) Darouassi Y, Righini CA, Reyt E. Mucoceles of the sphenoidal sinus: a report of four cases and review of the literature. B-ENT 2005;1:181-185.

(19) Chtioui-Saïed I. Les mucocèles des sinus de la face. A propos de 22 cas. Thèse Méd. Tunis, 2002. 